\title{
Management of traveller's diarrhoea with a combination of sodium butyrate, organic acids, and A-300 silicon dioxide
}

\author{
Lukasz Krokowicz' Jacek Mackiewicz ${ }^{2-4}$, Anna Wejman-Matela ${ }^{1}$, Piotr Krokowicz ${ }^{5}$, Michal Drews ${ }^{1}$, \\ Tomasz Banasiewicz ${ }^{1}$ \\ ${ }^{1}$ Chair and Department of General, Endocrinological, and Gastroenterological Oncology Surgery, Poznan University \\ of Medical Sciences, Poznan, Poland \\ ${ }^{2}$ Chair of Medical Biotechnology, Poznan University of Medical Sciences, Poznan, Poland \\ ${ }^{3}$ Department of Diagnostics and Cancer Immunology, Greater Poland Cancer Centre, Poznan, Poland \\ ${ }^{4}$ Department of Medical Oncology, Malgorzata Medical Centre, Srem, Poland \\ ${ }^{5}$ Department of General and Colorectal Surgery, Poznan University of Medical Sciences, Poznan, Poland
}

Prz Gastroenterol 2014; 9 (5): 285-290

DOI: $10.5114 /$ pg.2014.46164

Key words: traveller's diarrhoea, diarrhoea treatment, sodium butyrate, silicon dioxide, short-chain fatty acids.

\begin{abstract}
Address for correspondence: Lukasz Krokowicz MD, PhD, Chair and Department of General, Endocrinological, and Gastroenterological Oncology Surgery, Poznan University of Medical Sciences, 49 Przybyszewskiego St, 60-355 Poznan, Poland, phone: +48 618691122 , fax: +48 6186916 84, e-mail: Ikrokowicz@gmail.com
\end{abstract}

\begin{abstract}
Introduction: Traveller's diarrhoea (TD), defined by UNICEF/WHO as three or more unformed stools with or without other symptoms, imposes a considerable burden on travellers from developed countries. Various efforts have focused on decreasing the prevalence and severity of this condition.

Aim: To assess the efficacy of a combination of sodium butyrate, organic acids, and A-300 silicon dioxide in treatment providing symptomatic relief of TD.

Material and methods: The study was conducted in accordance with a protocol presented to the Bioethical committee of Poznan University of Medical Sciences. A total of 278 patients travelling to countries with higher risk of diarrhoea for at least 10 days were divided into a study arm being administered, in case of TD, a combination of sodium butyrate, organic acids, and A-300 silicon dioxide $(n=139)$ and a placebo arm $(n=139)$ with placebo administration.

Results: Forty-seven patients completed the study (22 in the study arm and 25 in the placebo arm). The diarrhoea occurrence after initiation of treatment at first symptoms was significantly lower in the study arm as compared to the placebo arm ( $9 \%$ vs. $36 \%, p=0.041$ ). Also, subjects from the study arm more frequently reported that the regimen administered had been efficient for their symptoms in comparison to the placebo arm $(72.7 \%$ vs. $32 \%, p=0.008)$. No adverse effects of the administered medication were noted during the study.

Conclusions: Sodium butyrate, organic acids, and A-300 silicon dioxide can be successful in decreasing symptoms of TD. Because of its efficacy and lack of observed side effects it has a strong potential in the treatment of patients with TD.
\end{abstract}

\section{Introduction}

Traveller's diarrhoea (TD) is a complex of clinical symptoms experienced by a traveller, with the presence of three or more unformed stools within $24 \mathrm{~h}$, commonly accompanied by abdominal cramps, nausea, vomiting, bloating, a sensation of faecal urge, fever, or blood in stools [1]. Similarly, according to the UNICEF/WHO definition, diagnosis of TD can be made with presence of three or more unformed stools within $24 \mathrm{~h}$, passed by a traveller with or without additional symptoms $[2,3]$. The presence of one or two loose stools a day is often defined as mild diarrhoea [1, 3, 4]. Traveller's diarrhoea has been assumed to be prevalent in $20-40 \%$ of travellers to destinations assumed to be high risk, such as Central America, South America, South Asia, and predominant parts of Africa, and less prevalent (10-15\%) in travellers to other destinations such as the Middle East, China, Russia, and southern Europe [5]. Groups of 
travellers at risk include not only tourists and travellers for business reasons but also soldiers during military and peacekeeping missions and pilgrims to sanctuaries located in the Middle East and India [6]. Geographical location of travel destination remains the single most important risk factor for acquiring TD. Many methods of TD treatment have proven helpful in reduction of TD symptoms, ranging from fluid replenishment to antibiotic treatment.

\section{Aim}

In our study we examined the role of sodium butyrate (SB), organic acids (short chain fatty acids - SCFA), and $\mathrm{A}-300$ silicon dioxide $\left(\mathrm{SiO}_{2}\right)$ in reduction of TD symptoms in comparison to placebo.

\section{Material and methods}

\section{Study group}

A total of 357 adult patients planning a trip to tropical countries were initially enrolled in the study. The countries of destination were Egypt, Tanzania, Tunisia, and Morocco. Upon initial office visit, the subjects were informed about the purpose of the study and accordance with the local Bioethical Committee and were examined medically. Upon the first visit 79 patients were excluded from the study for one of the following: active disease of the gastrointestinal tract, bacterial infection treated with an antibiotic within 14 days before the study, active immunosuppressive treatment, chemo- therapy, radiotherapy within the last 6 months, cachexia, active generalised disease, and an inability to comprehend the nature of study and treatment regimen. The remaining 278 adult patients signed the formal consent for the study, agreed to the daily oral treatment regimen, and collected the questionnaire, thus meeting the formal criteria of inclusion in the study. Patients were then randomised into a study arm and a placebo arm. The studied patients were provided with health insurance for the planned trip. Patients were instructed to record any potential side effects of administered therapy, and discontinue treatment when side effects were suspected. Of the randomised patients, 231 were excluded because of lack of the symptoms of travel disease or not returning the study questionnaire (complete information was obtained from 164 patients). A total of 47 patients with symptoms of travel disease, who returned the trip questionnaires, successfully completed the study (22 study, 25 placebo). The two study arms were found to be similar in age and gender. A flowchart regarding the course of randomised study is presented in Figure 1.

\section{Administered substance and regimen}

The patients from the study arm were administered a combination of sodium butyrate $(600 \mathrm{mg}$ ) encapsulated in a triglyceride matrix along with other components protected by triglyceride matrix: fumarate $(400 \mathrm{mg})$, citric acid (240 mg), malic acid (160 mg), sorbic acid

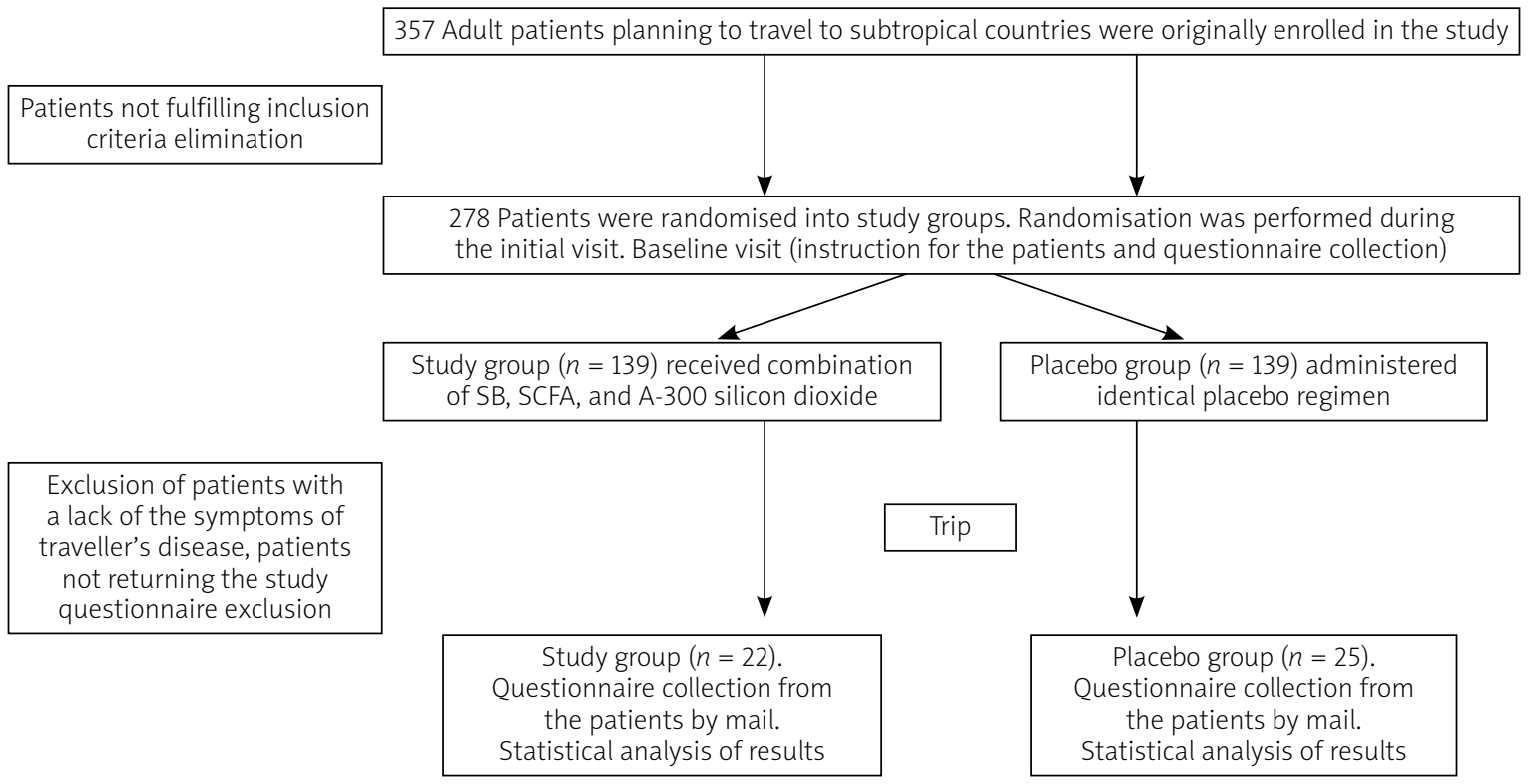

Figure 1. A flowchart representing the course of the randomised double-blinded clinical study of a combination of sodium butyrate and organic acids and A-300 silicon dioxide in the management of travellers' diarrhoea 
(200 mg), and A-300 silicon dioxide (1000 mg). This regimen was administered orally twice daily. Patients from the placebo arm were administered an identical regimen of placebo. Patients were advised to administer the medication in case of diarrhoea occurrence.

\section{Outcome assessment}

In order to assess the results of the study the participants of both arms were asked to complete records daily in a previously distributed questionnaire. The details regarding questionnaire records were explained to the participants upon the first visit before the trip. Patients were required to return the completed questionnaires by mail. The questionnaire recorded the observed occurrence of clinical symptoms associated with diarrhoea: the amount of stools per day, stool consistency, presence of blood, pus, or mucous in stool, abdominal pain, bloating, nausea, vomiting, elevated body temperature, and need of medical assistance or treatment during the trip. Based on these findings we sought the diagnosis of TD, which was defined as three or more unformed stools in $24 \mathrm{~h}$, passed by a traveller, commonly accompanied by abdominal cramps, nausea, and bloating, as defined by Hill [3].

\section{Statistical analysis}

The estimated standard deviation (SD) of the study was 1.2 with a confidence interval of 0.05 (two-tailed). With the control group of 20 subjects and the power of the test at 0.95, the number of cases in both arms was estimated for 20-30 participants. The amount of subjects completing the study fulfilled these criteria.

As the obtained results were of equal distribution, the results were analysed with a non-parametric test (Kruskal-Wallis test) or a Spearman correlation. The statistical significance was set at $p<0.05$. The size of samples and the power of the test were analysed with GraphPad StatMate 2.0 software (Graphpad, USA). Statistical analysis was performed with Statistica v.9.1 software (Statsoft Inc., USA).

\section{Results}

From the 357 patients who volunteered for the study a total of 278 patients qualified for the trial; 139 were qualified for the study arm, and 139 were qualified for the placebo arm. Finally, 22 patients in the study arm and 25 patients from the placebo arm completed the study. Nine percent of the patients in the study arm reported symptoms of diarrhoea developing after having administered the medication, as opposed to only $36 \%$ of patients from the placebo arm, which proved to be statistically significant $(p=0.041)$. Statistical significance was also observed ( $p=0.008)$ with regards to a subjective impression of medication being efficacious for the symptoms: $72.7 \%$ of patients from the study group noted that the medication administered was efficacious, as opposed to $32 \%$ of patients from the placebo group. Other investigated variables favoured the study group over the placebo arm but did not prove to be statistically significant: occurrence of three or more stools a day ( $4.5 \%$ vs. $28 \%$ ), subjective impression of symptoms negatively impairing the trip (91\% vs. 98\%), seeking medical help during the trip for diarrhoea symptoms (4.5\% vs. $8 \%$ ), subjective impression of the presence of mucus, pus, or blood in stool (4.5\% vs. $12 \%$ ), and continued symptoms after returning home (4.5\% vs. $16 \%$ ) (Table I).

\section{Discussion}

Available treatments and medications against TD are numerous. Each group of medications available has limitations regarding the efficacy, high cost, or inherent side effects. Efforts are constantly being made to investigate new therapies regarding TD.

In our previous study we revealed that administration of SB and SCFA for the prevention of traveller's diarrhoea is associated with a significant decrease of its symptoms. In the current study we sought potential in treatment of diarrhoea with a mixture of SB, SCFA, and A-300 silicon dioxide. We examined the frequency of symptoms and rates of medically diagnosed TD in a randomised placebo controlled study. To the best of our knowledge, this represents the first study of the role of SB, SCFA, and $\mathrm{A}-300 \mathrm{SiO}_{2}$ in the treatment of diarrhoea in travellers.

In the current placebo controlled randomised prospective trial we were able to demonstrate the efficacy of a combination of sodium butyrate, organic acids, and A-300 silicon dioxide against ongoing TD. The diarrhoea occurrence measured as a development of disease after first symptoms and initiation of treatment was significantly lower in the study arm as compared to the placebo arm ( $9 \%$ vs. $36 \%, p=0.041$ ). Subjects from the study arm also more frequently reported that the administered regimen had been efficient in reducing their symptoms, in comparison to the placebo arm $(72.7 \%$ vs. $32 \%, p=0.008)$. A trend suggesting the efficacy of the studied medication regimen over placebo was noted in such categories as occurrence of three or more stools a day, seeking less medical help for TD symptoms, subjective impression of less pus, blood, and mucus in stool, as well as fewer patients reporting diarrhoea symptoms after returning home; these, however, did not achieve statistical significance. There were no adverse effects noted within the study, either in the study arm or the placebo arm. 
Table I. Results of the study for study arm and placebo arm

\begin{tabular}{|c|c|c|c|c|c|}
\hline Variable & Study arm & Placebo arm & OR & $95 \% \mathrm{Cl}$ & Value of $p$ \\
\hline Patients who qualified for the study, $n$ & 139 & 139 & & & \\
\hline Patients who completed the study, $n$ & 22 & 25 & & & \\
\hline $\begin{array}{l}\text { Diarrhoea occurrence (development of disease } \\
\text { after first symptoms and treatment) (\%) }\end{array}$ & 9 & 36 & 0.18 & $0.03-0.94$ & 0.041 \\
\hline$\geq 3$ stools a day (\%) & 4.5 & 28 & 0.12 & $0.01-1.09$ & 0.0516 \\
\hline $\begin{array}{l}\text { Subjective impression of symptoms negatively } \\
\text { impairing the trip (\%) }\end{array}$ & 91 & 98 & 4.71 & $0.88-25.22$ & 0.0786 \\
\hline Medical help sought for diarrhoea symptoms (\%) & 4.5 & 8 & 0.22 & $0.02-2.03$ & 1.00 \\
\hline $\begin{array}{l}\text { Subjective impression of medication being } \\
\text { efficacious (\%) }\end{array}$ & 72.7 & 32 & 5.67 & $1.61-19.97$ & 0.0084 \\
\hline $\begin{array}{l}\text { Subjective impression of presence of mucus, pus, } \\
\text { or blood in stool (\%) }\end{array}$ & 4.5 & 12 & 0.35 & $0.03-3.63$ & 0.6115 \\
\hline $\mathrm{T}>37^{\circ} \mathrm{C}(\%)$ & 13.5 & 12 & 1.16 & $0.21-6.43$ & 1.00 \\
\hline Symptoms after return home (\%) & 4.5 & 16 & 0.25 & $0.03-2.43$ & 0.3525 \\
\hline $\begin{array}{l}\text { Subjective impression of symptoms negatively } \\
\text { impairing the trip - mild degree (\%) }\end{array}$ & 4.5 & 24 & 0.15 & $0.02-1.37$ & 0.102 \\
\hline $\begin{array}{l}\text { Subjective impression of symptoms negatively } \\
\text { impairing the trip - severe degree (\%) }\end{array}$ & 4.5 & 8 & 0.55 & $0.05-6.49$ & 1.00 \\
\hline
\end{tabular}

Sodium butyrate and organic acids such as fumarate, citric acid, sorbic acid, and malic acid constitute a medication group that has been known to act within the intestinal tract by facilitating alimentation of the intestinal mucosa, increasing intestinal blood flow, and stimulating peristalsis [7-9]. By providing nutrition to saprophytic intestinal bacteria it helps maintain the proper composition of intestinal bacterial flora, thus preventing absorption of toxins and development of harmful bacteria [10-24]. The presence of bacteria-producing short-chain fatty acids has been found to inhibit Escherichia coli, Campylobacter and Salmonella species both in the mechanism of competitive colonisation and antibacterial molecule secretion with immunological stimulation. The protective properties of butyrate also include stimulation of saprophytic bacterial flora, reduction in pathogenic adhesion to colonic wall, activation of the immunological system, including macrophages and mast cells in tissue, maintenance of adequate intestinal $\mathrm{pH}$, as well as anti-inflammatory and anti-oxidant properties $[25,26]$. In the case of diverticulitis exacerbation, it is the antibacterial properties of butyrate that have been associated with improvement of symptoms [13-15]. Short-chain fatty acids, including butyric acid, have the capacity to stimulate reabsorption of sodium and water in the intestine by stimulating the activity of ion pumps $\mathrm{Na}^{+} / \mathrm{H}^{-}$in epithelial cell membrane $[27,28]$. Animal models infected with cholera toxin showed significant efficacy of butyrate in the reduction of intestinal loss of water, sodium, and potassium chloride [29]. The use of butyrate in the case of diarrhoea also reduces inflammation, which reduces the duration of diarrhoea and reduces its intensity [30,31].

Decreasing production of butyric acid in the colon may cause serious problems in the elderly. It may expose them suffering traveller's diarrhoea after contact with a small number of antigens. The lack of a natural protective mechanism may cause much worse course of TD and consequences such as weakness, dehydration, etc. A declining amount of butyric acid in the colon can reduce the regenerative capacity of mucosa and its defence system.

With regard to the A-300 silicon dioxide, which was incorporated in the investigated regimen, it has been reported to be efficient in the treatment of diarrhoea and digestive tract infections and is primarily known as the active component of popular diosmectite. It lacks direct bactericidal or bacteriostatic properties and acts by creating a protective layer in the intestine by interacting with mucosal glycoproteins, improving mucosal layer durability against irritants [22, 32]. It can increase its resorptive surface and eradicate harmful substances, which is particularly efficient when used with simultaneous administration of substances providing nutrition to natural bacterial flora of the intestine [16-19].

Silicon dioxide $\left(\mathrm{SiO}_{2}\right)$ in combination with other mechanisms may have bactericidal and bacteriostatic effects. Silicon dioxide can act as a stimulant for macro- 
phages, leading to the disintegration of cells. It may also influence the tertiary structure of proteins causing the unveiling of the active centre, and it can stimulate the excessive production of reactive oxygen species (ROS).

The strong antibacterial action of $\mathrm{SiO}_{2}$ results from the formation of reactive oxygen species (ROS) that lead to lysis of bacteria. This may happen only in specific conditions. Bacteria are unable to take part in phagocytosis due to their inability to invaginate the cell wall. Bacteria secrete digestive enzymes that break down proteins, sugars, and fats. Then bacteria, on the basis of active transport using proteins located on the surface of the cell membrane, carry monosaccharides, amino acids, and short-chain fatty acids to the interior of its cells. Infiltration of silicon dioxide or other substances to the interior of the bacterial cell is very difficult. In vitro studies did not reveal any bacteriostatic or bactericidal effect of silicon dioxide on cultures as there is no mechanism that could enter these particles inside the bacterial cells. When there is a factor that allows silicon dioxide to penetrate into the bacterial cell due to NADPH oxidase, excess formation of ROS causes destruction of bacteria. The use of silicon dioxide as boosters of various drugs is becoming very reasonable [33-35].

Similarly to previous publications, the hypothesis of the current study assumed that in a balanced intestinal environment there are adequate levels of endogenic sodium butyrate needed for the wellbeing and regeneration of intestinal endothelium, but in a state of gastrointestinal infection and TD the observed decreased levels of endogenic butyrate measured in stools contribute to a degree of symptoms. Previously proven efficacy of this regimen against intestinal disease and lack of side effects has prompted the initiation of the current study in ongoing TD. This time, short-chain fatty acids, including sodium butyrate and A-300 silicon dioxide, were combined with the latter for improved pathogenic resorption.

Our placebo-controlled randomised study proved that administration of SB with SCFA and $\mathrm{SiO}_{2}$ may give very good effects in the treatment of TD. A significant decrease in the number of stools per day and a decrease in gastrointestinal symptoms shows that this might be a practical tool in the treatment of TD, especially in older people and patients suffering from other intestinal disorders.

However, the current study is not without its limitations. It would be of benefit to confirm the presented regiment of sodium butyrate, organic acids, and A-300 silicon dioxide in a multi-institutional randomised trial involving larger numbers of patients. Also a hypothesis of the synergistic properties of the current regimen for symptoms of ongoing TD has been assumed. It would require a separate dedicated study comparing the efficacy of A-300 silicon dioxide and sodium butyrate with fatty acids alone and the currently investigated combined regimen of the above.

\section{Conclusions}

The results of the current placebo-controlled prospective randomised study reveal that patients utilising a combination sodium butyrate, organic acids, and A-300 silicon dioxide in comparison to the placebo group noted an improvement in the symptoms of TD. No side effects were noted with utilisation of sodium butyrate, organic acids, and A-300 silicon dioxide. A combination of sodium butyrate, organic acids, and A-300 silicon dioxide can constitute an emerging direction in the treatment of patients with ongoing TD.

\section{Acknowledgments}

This study was funded by Mifarmex. The study sponsor had no role in the study design, collection, analysis, or interpretation of data, or in the writing of the report.

\section{References}

1. Passaro DJ, Parsonnet J. Advances in the prevention and management of traveler's diarrhea. Curr Clin Top Infect Dis 1998; 18: 217-36.

2. Diarrhoeal disease [http://www.who.int/mediacentre/factsheets/fs330/en/index.html].

3. Hill DR. Occurrence and self-treatment of diarrhea in a large cohort of Americans traveling to developing countries. Am J Trop Med Hyg 2000; 62: 585-9.

4. Al-Abri SS, Beeching NJ, Nye FJ. Traveller's diarrhoea. Lancet Infect Dis 2005; 5: 349-60.

5. Bourne S, Petrie C. The management of acute diarrhoea in a healthy adult population deploying on military operations. JR Army Med Corps 2008; 154: 163-7.

6. Ahmed QA, Arabi YM, Memish ZA. Health risks at the Hajj. Lancet 2006; 367: 1008-15.

7. Mortensen PB, Clausen MR. Short-chain fatty acids in the human colon: relation to gastrointestinal health and disease. Scand J Gastroenterol Suppl 1996; 216: 132-48.

8. Tonelli F, Dolara P, Batignani G, et al. Effects of short chain fatty acids on mucosal proliferation and inflammation of ileal pouches in patients with ulcerative colitis and familial polyposis. Dis Colon Rectum 1995; 38: 974-8.

9. Vernia P, Annese V, Bresci G, et al. Gruppo: Italiano per lo Studio del Colon and del Retto. Topical butyrate improves efficacy of 5-ASA in refractory distal ulcerative colitis: results of a multicentre trial. Eur J Clin Invest 2003; 33: 244-8.

10. Raqib R, Sarker P, Mily A, et al. Efficacy of sodium butyrate adjunct therapy in shigellosis: a randomized, double-blind, placebo-controlled clinical trial. BMC Infect Dis 2012; 12: 111.

11. Wedenoja S, Holmberg C, Höglund P. Oral butyrate in treatment of congenital chloride diarrhea. Am J Gastroenterol 2008; 103: 252-4. 
12. Song M, Xia B, Li J. Effects of topical treatment of sodium butyrate and 5-aminosalicylic acid on expression of trefoil factor 3 interleukin 1 beta, and nuclear factor kappaB in trinitrobenzene sulphonic acid induced colitis in rats. Postgrad Med J 2006; 82: 130-5.

13. Lawrance IC. Novel topical therapies for distal colitis. World J Gastrointest Pharmacol Ther 2010; 1: 87-93.

14. Bloemen JG, Schreinemacher MH, de Bruine AP, et al. Butyrate enemas improve intestinal anastomotic strength in a rat model. Dis Colon Rectum 2010; 53: 1069-75.

15. Hamer HM, Jonkers DM, Bast A, et al. Butyrate modulates oxidative stress in the colonic mucosa of healthy humans. Clin Nutr 2009; 28: 88-93.

16. Dushkin AV, Gaidul' KV, Gol'dina IA, et al. Antimicrobial activity of mechanochemically synthesized composites of antibiotics and nanostructured silicon dioxide. Dokl Biochem Biophys 2012; 443: 61-3.

17. Kim WJ, Ahn SY, Kim JH, et al. Synthesis and size characterization of silica nanospheres using sedimentation field-flow fractionation (SdFFF). J Nowosci Nanotechnol 2012; 12: 610-7.

18. Hrenovic J, Milenkovic J, Ivankovic T, et al. Antibacterial activity of heavy metal-loaded natural zeolite. J Hazard Mater 2012; 201-202: 260-4

19. Gu G, Xu J, Wu Y, et al. Synthesis and antibacterial property of hollow $\mathrm{SiO} 2 / \mathrm{Ag}$ nanocomposite spheres. J Colloid Interface Sci 2011; 359: 327-33.

20. Banasiewicz T, Krokowicz L, Stojcev Z, et al. Microencapsulated sodium butyrate reduces the frequency of abdominal pain in patients with irritable bowel syndrome. Colorectal Dis 2013; 15: 204-9.

21. Krokowicz L, Kaczmarek BF, Krokowicz P, et al. Sodium butyrate and short chain fatty acids in prevention of travellers' diarrhoea. A randomized prospective study. Travel Med Infect Dis 2014; 12: 183-8.

22. Guarino A, Lo Vecchio A, Pirozzi MR. Clinical role of diosmectite in the management of diarrhea. Expert Opin Drug Metab Toxicol 2009; 5: 433-40.

23. Andoh A, Bamba T, Sasaki M. Physiological and anti-inflammatory roles of dietary fiber and butyrate in intestinal functions. JPEN J Parenter Enteral Nutr 1999; 23 (5 Suppl.): S70-3.

24. Rabbani GH, Albert MJ, Hamidur Rahman AS, et al. Short-chain fatty acids improve clinical, pathologic, and microbiologic features of experimental shigellosis. J Infect Dis 1999; 179: 390-7.

25. Claus R, Günthner D, Letzguss H. Effects of feeding fat-coated butyrate on mucosal morphology and function in the small intestine of the pig. J Anim Physiol Anim Nutr (Berl) 2007; 91: 312-8.

26. Dianzani C, Cavalli R, Zara GP, et al. Cholesteryl butyrate solid lipid nanoparticles inhibit adhesion of human neutrophils to endothelial cells. Br J Pharmacol 2006; 148: 648-56.

27. Senagore AJ, MacKeigan JM, Scheider M, Ebrom JS. Shortchain fatty acid enemas: a cost-effective alternative in the treatment of nonspecific proctosigmoiditis. Dis Colon Rectum 1992; 35: 923-7.

28. Lührs H, Gerke T, Müller JG, et al. Butyrate inhibits NF-kappaB activation in lamina propria macrophages of patients with ulcerative colitis. Scand J Gastroenterol 2002; 37: 458-66.
29. Vernia P, Annese V, Bresci G, et al.; Gruppo Italiano per lo Studio del Colon and del Retto. Topical butyrate improves efficacy of 5-ASA in refractory distal ulcerative colitis: results of a multicentre trial. Eur J Clin Invest 2003; 33: 244-8.

30. Rabbani GH, Albert MJ, Rahman H, Chowdhury AK. Short-chain fatty acids inhibit fluid and electrolyte loss induced by cholera toxin in proximal colon of rabbit in vivo. Dig Dis Sci 1999; 44: 1547-53.

31. Banasiewicz T, Borycka-Kiciak K, Dobrowolska-Zachwieja A, et al. Clinical aspects of sodium butyrate application in dietary treatment of bowel diseases [Polish]. Prz Gastroenterol 2010; 5: 329-34.

32. Dupont C, Vernisse B. Anti-diarrheal effects of diosmectite in the treatment of acute diarrhea in children: a review. Paediatr Drugs 2009; 11: 89-99.

33. Chu Z, Huang Y, Li L, et al. Physiological pathway of human cell damage induced by genotoxic crystalline silica nanoparticles. Biomaterials 2012; 33: 7540-6.

34. Farcal LR, Uboldi C, Mehn D, et al. Mechanisms of toxicity induced by $\mathrm{SiO} 2$ nanoparticles of in vitro human alveolar barrier: effects on cytokine production, oxidative stress induction, surfactant proteins A mRNA expression and nanoparticles uptake. Nanotoxicology 2013; 7: 1095-110.

35. Sandberg WJ, Lag M, Home JA, et al. Comparison of non-crystalline silica nanoparticles in IL-1beta release from macrophages. Part Fibre Toxicol 2012; 9: 32.

Received: 27.03 .2014

Accepted: 3.07 .2014 